\title{
METHODOLOGY FOR ESTIMATING THE INFLUENCE OF INTERACTION OF DEFECTS IN SHAFT PREPARATIONS
}

\author{
Nikolay KORIHIN, Daria KITAEVA \\ Peter the Great St.Petersburg Polytechnic University, St.Petersburg, Russia, dkitaeva@mail.ru
}

https://doi.org/10.37904/metal.2021.4148

\begin{abstract}
In power plants, special concerns from the point of view of destruction are caused by parts rotating at high frequencies, especially metal-consuming and highly loaded shafts of turbo-machines. When analyzing data on the interaction of the most dangerous metallurgical defects - cracks - the concept of "relative size" of a defect (RDS) is used, which is the ratio of half of the total size of defects to the distance between their centers. The available recommendations for problems of various classes are not always unambiguous, which leads to an overestimation of the stress intensity factor (SIF) by approximately 1.5 times. To assess the influence of interacting defects, it was proposed to use a specially obtained function, which is a correction function for determining the stress intensity factor and is determined experimentally using the photo-elasticity method. A special correction function (SPF) depends on the geometric parameters of the model, cracks and their location in the body of the model. In the case of pairs of defects of the same type and their identical sizes, the parameters of the SPF are a characteristic of the interaction between defects. The procedure for the photoelastic determination of the SIF is described in detail, and the experimental dependences of the SIF for the characteristic points of interacting defects are presented. Cases with the RDS from 0 to 0.77 were considered. The characteristic values are determined, which make it possible to obtain three ranges of RDS for internal and surface interacting defects. Experimental dependences of the SPF on the RDS are obtained for internal, surface, and surface-internal interacting defects.
\end{abstract}

Keywords: Photo-elasticity method, internal defect, surface defect, multiple defect, stress intensity factor

\section{INTRODUCTION}

Calculation and experimental methods male it possible to determine the degree of danger of single crack-like defects and take into account their influence in the calculations of parts of strength [1,2].

At the same time, the question of the accumulation of defects, when their interaction can enhance the negative effect on the structural strength of parts, has not been studied enough [3-6].

When analyzing data on the interaction of the most dangerous metallurgical defects - cracks - the concept of "relative size" of a defect $\lambda$ is used, which is the ratio of half of the total size of defects to the distance between their centers [7].

Currently, there are a number of recommendations for problems of various classes, according to which defects are considered to be interacting at $\lambda \geq 0.2 \ldots 0.5$, and combined - at $\lambda<0.2 \ldots 0.5$ [7-10].

In other words, the available recommendations are in rather wide range and are not always unambiguous. For example, in one work [8] it is proposed to combine round collinear cracks at $\lambda>0.67$, and in another [9] this recommendation is considered overly conservative. In the opinion of the authors of [9], the stress intensity factor (SIF) in this case is overestimated by approximately one and a half times, which is why they propose to consider the cracks united at $\lambda>0.9$. 


\section{ANALYSIS OF EXPERIMENTS}

The [9] details the method of photo-elastic definition of SIF (Figure 1) and provides experimental dependencies for points $A$ and $B$ of interacting defects (Figure 2). To assess the impact of the interacting defects, it is proposed to use the experimentally obtained function $f[10]$.

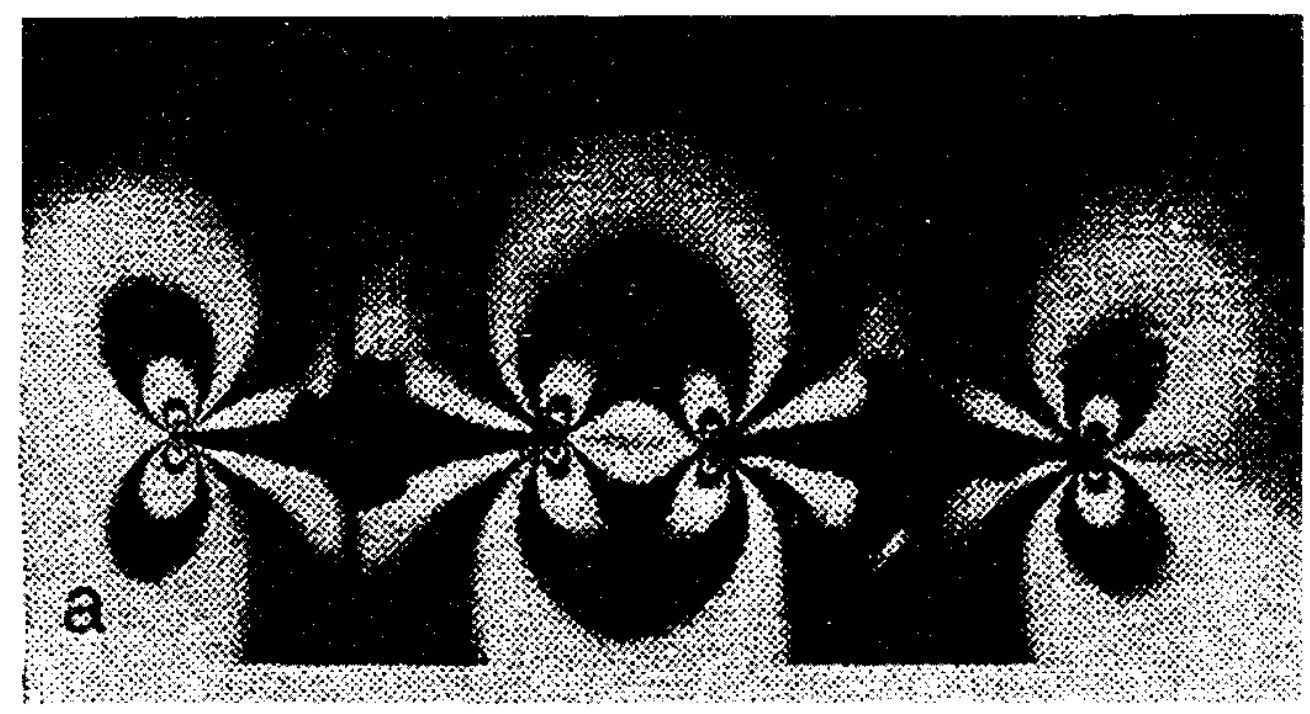

Figure 1 Isochromes on cuts from "frozen" when rotating models of shafts

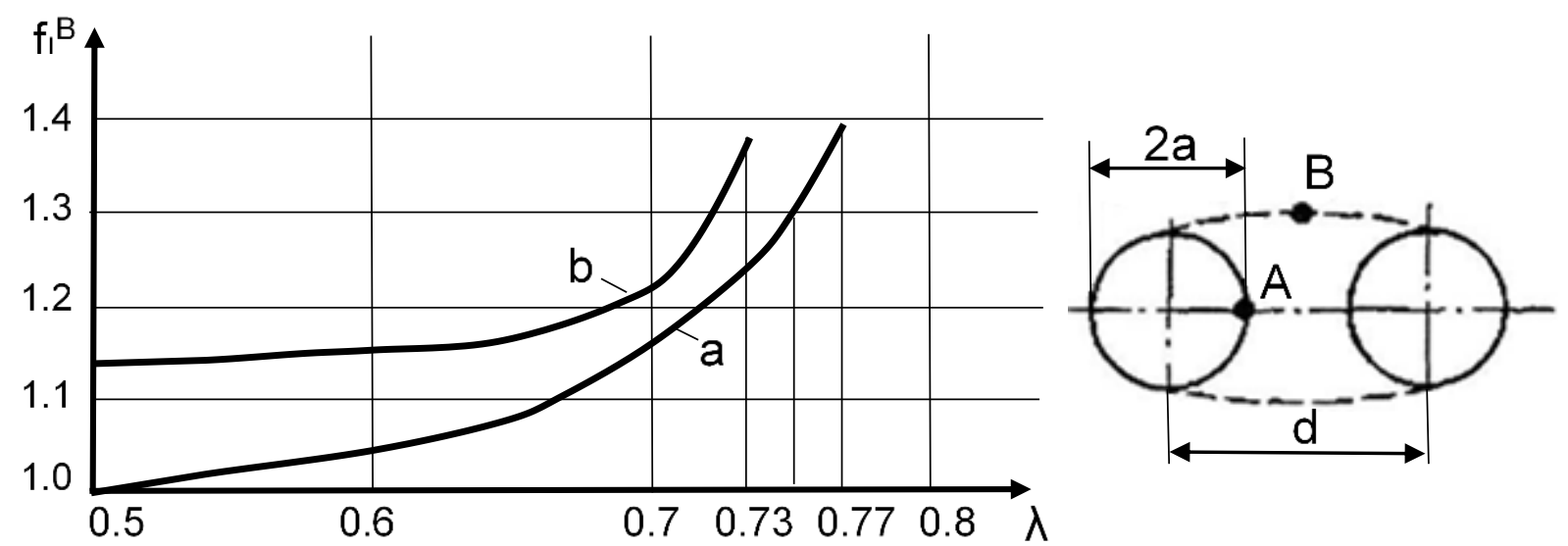

Figure 2 The effect of interaction: $a$ - for internal defects; $b$ - for surface defects

In a monotonous change of $\lambda$, the interaction processes between defects differ. Thus, with the increase of $\lambda$ from 0 to its some value $\lambda_{1}$, there is almost no interaction. The strength of the body in this case is determined by the size of the defects.

As the $\lambda$ increases (from $\lambda_{1}$ to some value $\lambda_{2}$ ) a noticeable interaction of defects happens. The strength of the body herewith depends on the size of the defects and the effects of interaction.

When $\lambda=\lambda_{2}$ the effects of the interaction become more intense and the strength of the body is determined not by the size of the initial defects and the effect of interaction, but by the size of the integral defect. According to the results of experiments obtained on epoxy samples and models of shafts of machines with similar types of defects, if the magnitude of the integral defect exceeds its critical size (for current stresses), after the destruction of the jumper there is a non-stop avalanche-like destruction of the sample or part. In other cases, 
when $\lambda>\lambda_{2} \ldots 1$, there is three-stage destruction: the destruction of the jumper, the rise of stresses to the critical value, the final fragile destruction.

\section{METHOD OF ASSESSING THE IMPACT OF DEFECT INTERACTION}

The maximum values of SIF of interacting defects, marked at point $A$, can be imagined as

$K_{I}^{A}(\lambda)=\sigma(\pi \cdot a)^{\frac{1}{2}} \cdot f_{I_{0}} \cdot f_{I}^{B}(\lambda)$,

where:

$\sigma-$ nominal stress

$a-$ round crack radius

$f_{I_{0}}$ - correction factor for a single round defect in infinite body

$f_{I}^{B}(\lambda)$ - correction factor on the effect of interaction between cracks at point $\mathrm{A}$

Correction factor $f_{I}^{B}(\lambda)$ is determined from the experimental data on the formula

$f_{I}^{B}(\lambda)=\frac{f_{I}^{B H}(\lambda)}{f}$,

where $\left(f=f_{I}^{H}\right.$ when $\left.\lambda \leq 0.3\right)$ (Figure 3).

According to the methodology [10], in our case, the value of $K_{l}$ at point A can also be presented with the help of a common dependency for an elliptical defect in an infinite plate of constant thickness $h$ on the formula

$K_{I}^{A}(\lambda)=\frac{1,13 \cdot \sigma \cdot a^{\frac{1}{2}}}{\left\{1-\lambda^{1,8}\left[0,6-\left(0,5-\frac{1}{8 \lambda}\right)^{2}\right]\right\}^{0,54}}$,

The maximum value of SIF on the contour of a newly formed integral defect takes place at point B [9]:

$K_{I}^{B}(\lambda)=\sigma\left(\pi \cdot a_{M}\right)^{\frac{1}{2}} \cdot f_{I_{0}} \cdot f_{M}(\lambda)$,

where:

$f_{M}(\lambda)$ - correction factor for the ellipticity of the defect at point $\mathrm{B}$

$a_{M}$ - radius of a round crack equivalent in an area to ellipse-shaped

The graphic dependence of $f_{M}(\lambda)$ is known [10]. In this case

$K_{I}^{B}(\lambda)=\sigma[\pi \cdot a \cdot(\pi \cdot a+2 d)]^{\frac{1}{4}} \cdot f_{I_{0}} \cdot f_{M}(\lambda)$.

The $K_{I}^{A}(\lambda)$ and $K_{I}^{B}(\lambda)$ values defined by dependencies (1), (3), (5) are listed in Figure 4 in relative coordinates. Where $K_{I_{0}}=\sigma(\pi \cdot a)^{1 / 2} \cdot f_{I_{0}}-$ SIF for single round crack.

The parameter $\lambda_{2}$ can be set from the condition $K_{I}^{A}(\lambda)=K_{I}^{B}(\lambda)$. This value is found based on the accuracy of the necessary assessments and the methods used. Thus, multiple defects, depending on the degree of their interaction, can be conditionally divided into single (when $\lambda<\lambda_{1}$ ), interacting (when $\lambda_{1} \leq \lambda \leq \lambda_{2}$ ) and combined (when $\lambda>\lambda_{2}$ ). 

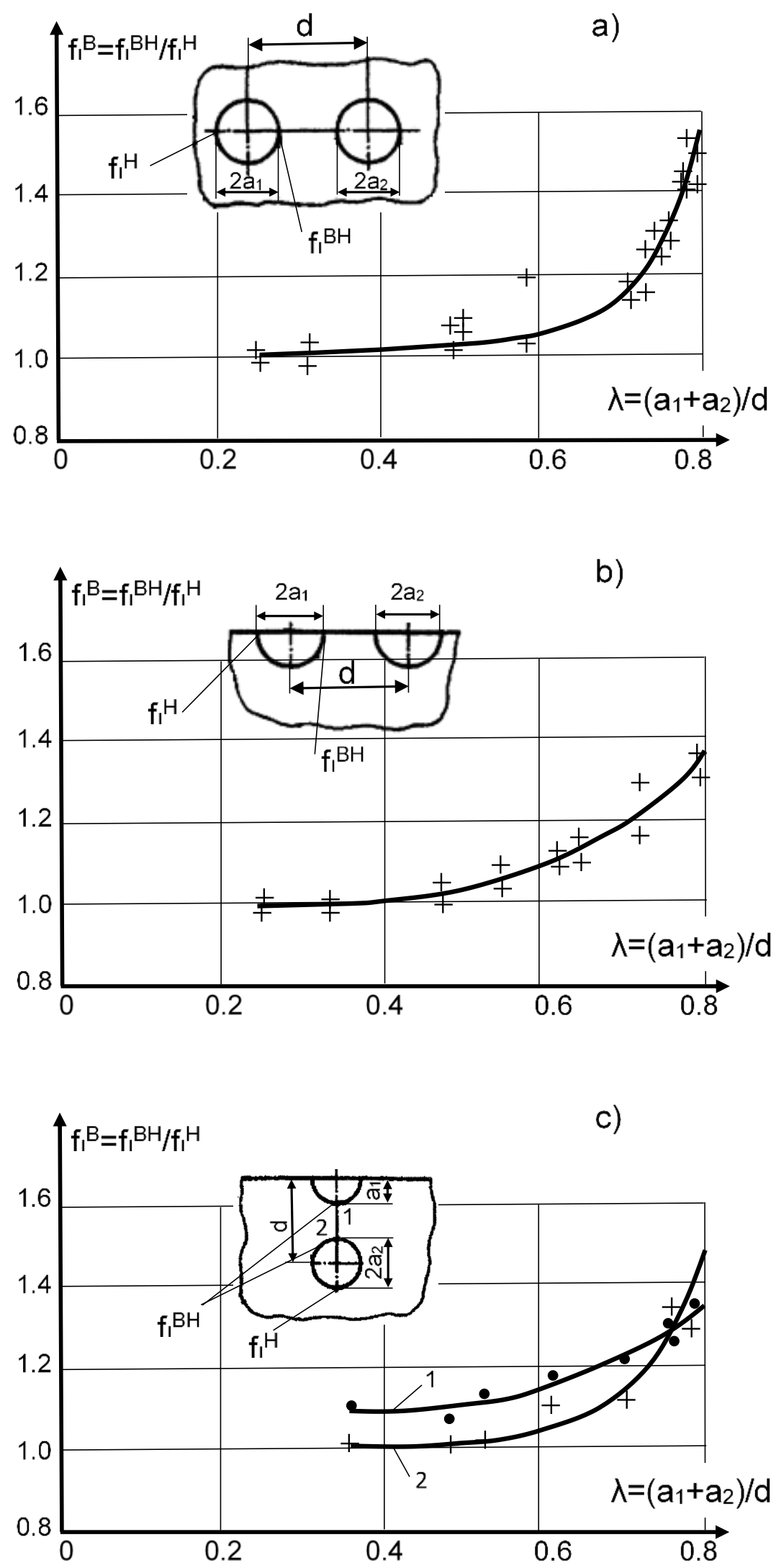

Figure 3 Interaction effect: $a$ - for internal defects; $b$ - for surface defects; $c$ - for surface-internal defects 


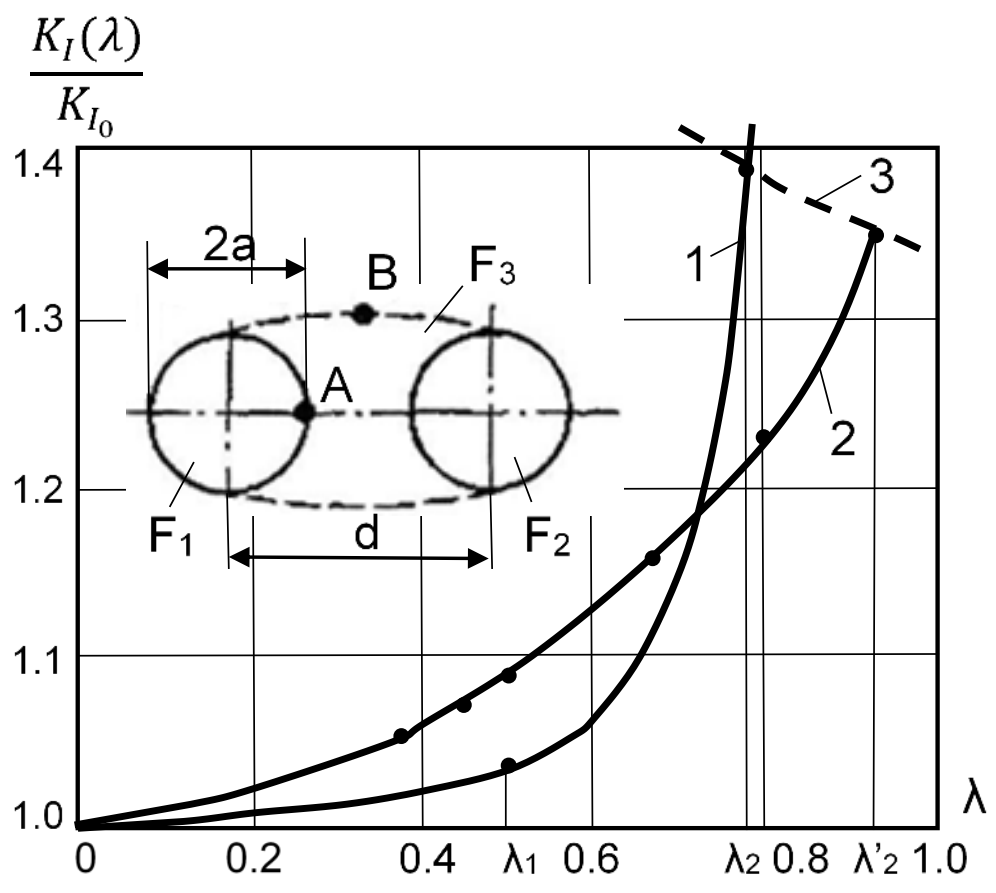

Figure 4 SIF dependencies at points $A$ (solid lines) and B (bar lines) from the relative distance between the internal distances. Line 1 - experiment, 2, 3 - according to the source [9].

\section{CONCLUSION}

Experimental data have shown that for internal defects $\lambda_{1}=0.5, \lambda_{2}=0.77$; when $0.5<\lambda<0.77$ correction factor $K_{I}^{B}(\lambda)$ can be defined from graphic dependence $a$ in Figure 2; when $\lambda<0.5 f_{I}^{B}=1$, when $\lambda>0.77$ $f_{I}^{B}=1.39$. For surface defects, similarly obtained $\lambda_{1}=0.5, \lambda_{2}=0.73$; when $<0.5 f_{I}^{B}=1.14$; when $0.5 \leq \lambda \leq$ $0.73 f_{I}^{B}$ can be found from graphic dependence $b$ in Figure 2, when $\lambda>0.73 f_{I}^{B}=1.385$. For surface-internal interacting defects, the effects of interaction $f_{I}^{B}$ and values of $\lambda_{1}, \lambda_{2}$ are within the limits set for superficial and internal defects.

\section{REFERENCES}

[1] MAKHUTOV, N.A. Strength of structural elements to brittle destruction. Moscow: Mashinostroenie, 1973.

[2] TITOV, V.B. Experimental method for determining the three types of stress intensity coefficients. In: Seminar "Interference-optical methods of the deformable solid mechanics and rock mechanics". Novosibirsk, 1985, pp. 8283.

[3] YUKAVA, S., TIMO, D.P., RUBKO, A.K. In Book: Destruction. Moscow: Mashinostroenie, 1977, vol. 5.

[4] VISWANATHAN, R., JAFFI, R.I. Metallurgical factors affecting the reliability of steam turbine rotors in organic fuel plants. Transactions of the American Society of Mechanical Engineers. 1985, vol. 107, no. 3, pp. 45-52.

[5] RUDAEV, Y., KITAEVA, D. On structural and phase transitions in aluminum alloys. Journal of Physics: Conference Series. 2018, vol. 1141, no. 1, pp. 012074.

[6] ARTIUKH, V., MAZUR, V., SAHIROV, Y., KAPUSTINA, N. Protection of Metallurgical Machines from Breakdowns at Iron and Steel Works. Advances in Intelligent Systems and Computing. 2020, vol. 1115 AISC, pp. 950-962.

[7] PANASYUK, V.V., SAVRUK, M.P., DATSISHIN, A.P. Stress distribution near cracks in plates and shells. Kiev: Naukova dumka, 1976. 
[8] SCHMITT, W., KEIN, E., WELLEIM, R., BARTHOLOME, G. Linear elastic stress intensity factors for cracks in nuclear pressure vessel nozzles under pressure and temperature loading. International Journal of Pressure Vessels and Piping. 1980, vol. 8, no. 1, pp. 89-96.

[9] MR108.7-86. Methodological recommendations. Power equipment. Calculations and strength tests. Calculation of stress intensity factors. Moscow: CNIITMASH, 1986.

[10] KORIHIN, N.V. Ensuring the strength of parts and assemblies of turbines using the photoelasticity method. St. Petersburg: Polytechnical Publishing House, 2008. 